\title{
Subpulmonary pleural effusions in children after cardiac surgery
}

\author{
CHRISTOPHER O’CALLAGHAN, „ JULIE COOKE, $\dagger$ IAN H KERR, $\dagger$ \\ ELLIOT A SHINEBOURNE*
}

$\star$ From the Department of Paediatric Cardiology, and †Department of Radiology, Brompton Hospital, London

SUMMARY Postoperative chest radiographs on 100 children who had undergone cardiac operations were evaluated to determine the frequency of subpulmonary effusions after surgery. Of the 83 patients in whom adequate erect postoperative radiographs were available, $9(11 \%)$ had effusions confirmed by lateral decubitus radiographs. On the frontal chest $x$ ray film, the evidence of an effusion was an increase in distance between the diaphragm and air in the gastric fundus, or an apparent elevation of the right hemidiaphragm. None of the patients examined echocardiographically had associated pericardial effusions. When a subpulmonary effusion was detected diuretic treatment was started or continued. None of the patients had radiographic evidence of residual fluid when they were seen two weeks after their discharge from hospital.

Pleural effusions are common and may be bilateral. ${ }^{1}$ When fluid collects in the chest it initially accumulates inferiorly between the lung and the diaphragm and posteriorly in the costophrenic sulcus. ${ }^{2}$ As the amount of fluid increases it spreads around the lung, which retracts towards the hilum. A typical effusion collects mainly laterally and posteriorly, obscures the hemidiaphragm and costophrenic sulcus, and has a smooth concave medial and lateral border.

Occasionally a large volume of fluid may collect between the lung and the diaphragm without obliterating the costophrenic sulcus. These effusions may escape detection on the radiograph unless the degree of suspicion is high. ${ }^{3}$ They should be suspected if one hemidiaphragm appears to be elevated or if the distance between the left hemidiaphragm and air in the gastric fundus is increased. Subpulmonary effusions have been reported in patients with nephrotic syndrome, ${ }^{45}$ metastatic cancer, ${ }^{6}$ congestive cardiac failure, and constrictive pericarditis. ${ }^{2}$

A missed subpulmonary effusion on the chest radiograph of a child who subsequently died of late tamponade prompted this study to determine the frequency of subpulmonary effusions in children after cardiac operations.

Requests for reprints to Dr E A Shinebourne, Department of Paediatric Cardiology, Brompton Hospital, Fulham Road, London SW3 6HP.

Accepted for publication 21 August 1985

\section{Patients and methods}

We studied 100 consecutive children undergoing cardiac operation at this hospital (Table). Their ages ranged from the neonatal period up to 14 years (mean age 3 years 2 months, median age 1 year 6 months).

All children had routine preoperative and supine postoperative radiographs while in the intensive care unit. Most children also had a routine erect chest radiograph on the day of discharge, or before if clinically indicated. Any child thought to have a subpulmonary effusion demonstrated by an increased distance between the gastric air bubble and the lung (Fig. 1a) or an apparent raised right dome of the diaphragm on the erect chest $x$ ray film (Fig. 2) had right or left lateral decubitus radiographs (Fig. 1b) to confirm the presence of an effusion. An echocardiographic examination was performed on all patients with a subpulmonary effusion to detect any associated pericardial fluid. Routine chest radiographs taken two weeks after discharge were also reviewed.

\section{Results}

Of the 100 children entered into the study, 10 were transferred to other hospitals and seven died in the intensive care unit without an erect chest radiograph being taken. A subpulmonary effusion was suspected in 17 of the remaining 83 children ( 13 on the 593 

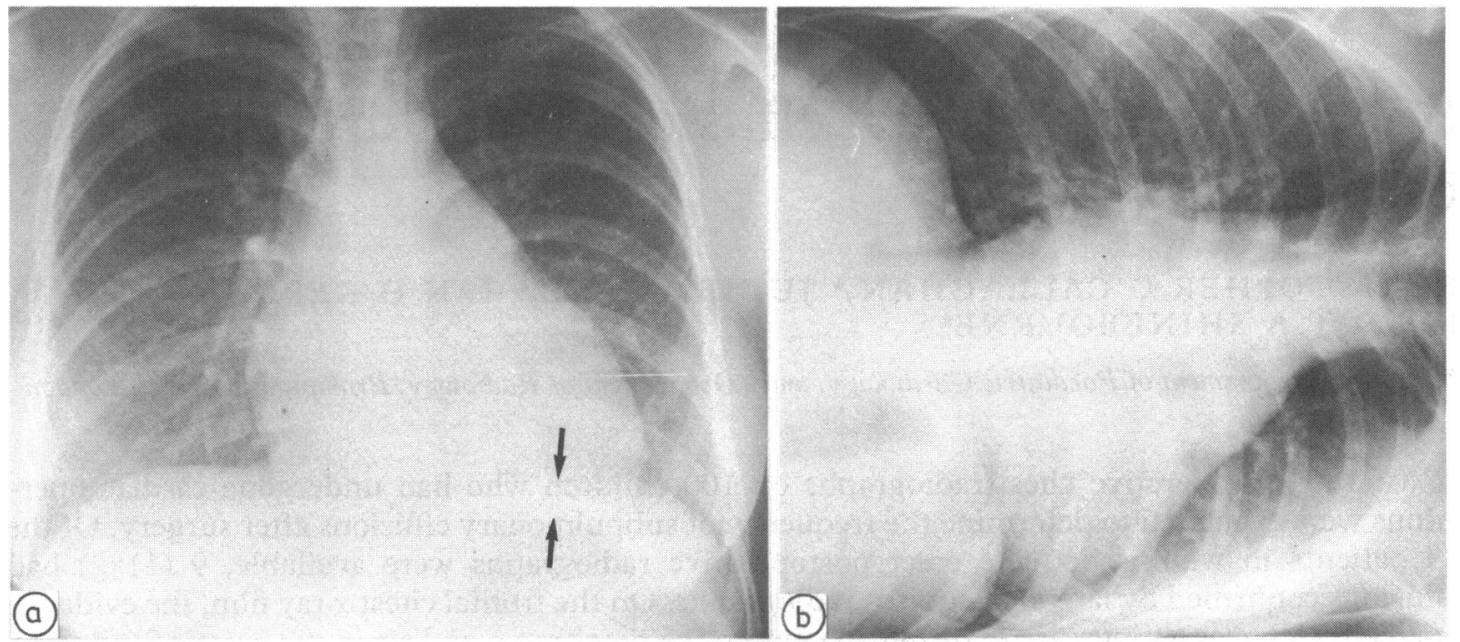

Fig. 1 (a) Erect radiograph showing increased distance between the diaphragm and the gastric air bubble. (b) Left lateral decubitus radiograph of the same patient showing a left sided pleural effusion.

left and four on the right). Effusions were excluded in eight and confirmed in nine children by a lateral decubitus film. All confirmed subpulmonary effusions were present on the left side.

None of the patients with subpulmonary effusions had an associated pericardial effusion detected by cross sectional echocardiography. Three children were not on regular postoperative diuretic treatment when their subpulmonary effusion was noted. There was no radiographic evidence of residual fluid in patients seen two weeks after discharge from hospital.

\section{Discussion}

The radiological signs of a subpulmonary effusion are subtle and may be missed. ${ }^{2}$ A subpulmonary Table Operations performed on 83 children

\begin{tabular}{lrl}
\hline Operation & No & $\begin{array}{l}\text { Subpulmonary } \\
\text { effusion }\end{array}$ \\
\hline Requiring cardiopulmonary bypass: & & \\
Ventricular septal defect & 15 & 3 \\
Tetralogy of Fallot & 11 & \\
Mustard operation & 7 & \\
Atrial septal defect & 5 & 2 \\
Aortic valvotomy & 4 & \\
Atrioventricular canal defect & 4 & \\
Anatomical switch for & & \\
transposition of the great & 4 & \\
vessels & 3 & 1 \\
Fontan procedure & 2 & \\
Mitral valve repair & 1 & \\
Rastelli operation & 1 & \\
Pulmonary valvotomy & 6 & \\
Not requiring cardiopulmonary bypass: & 6 \\
Coarctation repair & 6 & 2 \\
Pulmonary artery band & 5 & \\
Left Blalock operation & 5 & 1 \\
Patent ductus arteriosus ligation & 5 & \\
Right Blalock operation & 4 & \\
\hline
\end{tabular}

effusion on the left side increases the distance between the air in the gastric fundus and the diaphragm. On the right side the only sign may be a slight elevation of the hemidiaphragm. Occasionally, there is blunting of the lateral costophrenic sulcus (that is loss of the normal acute angle between thoracic cage and diaphragm) and a small lamellar effusion may be seen along the lateral chest wall. When the patient is placed on the side, the fluid is free to move within the chest and collects along the lateral chest wall. Fluid in this position is readily detected by a horizontal ray lateral decubitus radiograph (Fig. 1b). A large effusion may be missed on a frontal radiograph if the above signs are not recognised.

There is some controversy about the origin of subpulmonary effusions. Perhaps the most plausible explanation was put forward by Fleischner. ${ }^{2} \mathrm{He}$

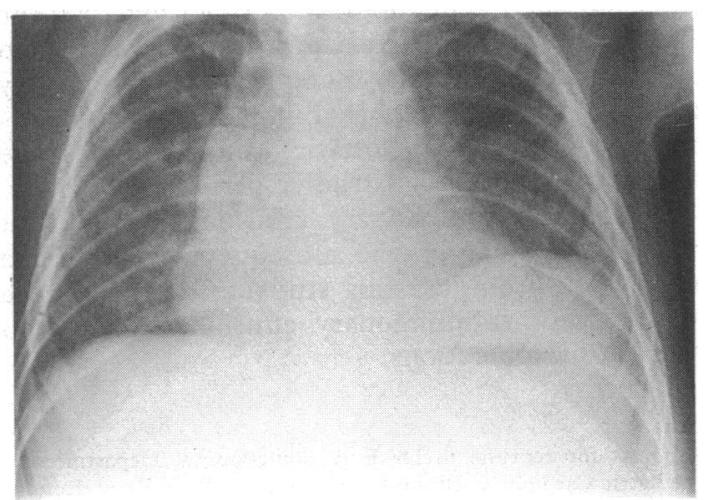

Fig. 2 Erect radiograph showing elevation of the left diaphragm. 
stated that recumbency after surgery increases the tendency to lower lobe atelectasis, elevation of the diaphragm, and reduced cough. The lower lobe retracts as soon as a pleural effusion appears. This additional atelectatic pull increases the amount of fluid held between the lower lobe and diaphragm.

In our study $11 \%$ of children had a subpulmonary effusion. Their occurrence only on the left side may be explained by the fact that most operations are performed via a central or left thoracotomy incision. In one patient, however, a right thoracotomy for a modified Blalock-Taussig operation produced a left sided subpulmonary effusion. It is of interest that three of the nine children with effusions were not receiving diuretics, which we usually prescribe after cardiac surgery.

In conclusion, we found an unexpectedly high number of subpulmonary effusions after cardiac surgery in children. Diagnosis may be suspected after careful examination of an erect chest radio- graph and can be confirmed by examination of a chest radiograph taken from the appropriate lateral decubitus view. This high frequency of effusion confirms the need for routine postoperative diuretics after cardiac surgery.

\section{References}

1 Ochsner SF, Coles CC. Bilateral subpulmonary effusion. I. Easy method for detection. $₹ \mathrm{La}$ State Med Soc 1976; 128: 9-10.

2 Fleischner FG. Atypical arrangement of free pleural effusion. Radiol Clin North Am 1963; 1: 347-62.

3 Petersen JA. Recognition of infrapulmonary pleural effusions. Radiology 1960; 74: 34-41.

4 Barry WF Jr. Infrapulmonary pleural effusion. Radiology 1956; 66: 740-3.

5 Dunbar JS, Favreau M. Infrapulmonary pleural effusion with particular reference to its occurrence in nephrosis. f Can Assoc Radiol 1959; 10: 24-33.

6 Friedman RL. Infrapulmonary pleural effusions. $A \mathcal{F}$ 1954; 71: 613-23. 